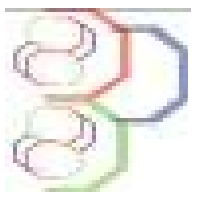

\title{
Les plantes médicinales utilisées contre les dermatoses dans la pharmacopée Baïnounk de Djibonker, région de Ziguinchor (Sénégal).
}

\author{
DIATTA Cheikh Daouda1*, GUEYE Mathieu² et AKPO Léonard Elie ${ }^{3}$

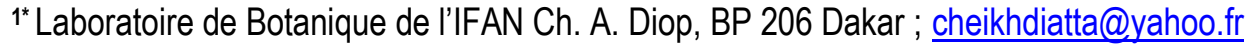 \\ ${ }^{2}$ Laboratoire de Botanique de l'IFAN Ch. A. Diop\& UMI 3189, BP 206 Dakar ; queye_quirane@yahoo.fr \\ 3 Département de Biologie végétale, Faculté des Sciences et Techniques, UCAD, BP 5005 Dakar \\ ${ }^{1 *}$ Correspondant : Tél : 00 (221) 7751644 46, courriel : cheikhdiatta@yahoo.fr
}

Original submitted in on $6^{\text {th }}$ September Published online at www.m.elewa.org on $31^{\text {st }}$ October 2013.

https://dx.doi.org/10.4314/jab.v70i1.98762

\section{RESUME}

Objectifs : Cette étude a pour objectif de connaitre les plantes médicinales utilisées comme remède dans le traitement des dermatoses dans la pharmacopée Baïnounk de Djibonker, région de Ziguinchor (Sénégal).

Méthodes et résultats : La technique des entretiens ouverts semi structurés est utilisée pour recueillir les informations. L'étude a permis de recenser trente-six espèces traditionnellement utilisées dans le traitement des dermatoses. Elles sont réparties en vingt-cinq familles et trente-six genres. Les familles les mieux représentées sont les Apocynaceae, les Papilionaceae et les Rubiaceae avec chacune trois espèces. Les plantes utilisées sont surtout des arbres (36\%) et des arbustes (30\%). Les espèces les plus indiquées sont Khaya senegalensis (94,4\%), New bouldia laevis (88,9\%), Carapa spp et Solanum cerasiferum $(77,8 \%$ chacune). Les feuilles sont les organes les plus utilisés avec $46 \%$ suivis des racines avec $21 \%$. La macération $(40 \%)$ et le broyage (27\%) sont les modes de préparation les plus courant. Les maladies liées à la peau (78\%) sont les plus fréquentes, suivies de celles de la muqueuse (12\%).L'administration percutanée (44\%) est la plus utilisée puis par bain $(21 \%)$.

Conclusion et applicabilité des résultats : Ces résultats révèlent que les plantes médicinales jouent un rôle très important dans le traitement des dermatoses et leur valorisation nécessite véritablement l'apport des savoirs locaux qui sont détenues par les populations rurales.

Mots clés : Ethnobotanique, Dermatoses, Plantes médicinales, Pharmacopée sénégalaise, Bainounk.

\begin{abstract}
Objectives: To know the medicinal plants used in the treatment of skin diseases in the pharmacopoeia of Bainounk of Djibonker, Ziguinchor region (Senegal).

Methods and results: The technique of open semi-structured interviews was used to collect information. The study has identified thirty-six species traditionally used in the treatment of skin diseases. They are divided into twenty-five families and thirty-six genera. The best represented families Apocynaceae, Papilionaceae and Rubiaceae with each three species. The plants used are mainly trees (36\%) and shrubs (30\%). The most appropriate species Khaya senegalensis (94.4\%), New bouldia laevis (88.9\%), Carapa spp. and Solanum
\end{abstract}


cerasiferum (77.8\% each). The leaves are the organs most used with $46 \%$ come from the roots with $21 \%$. Maceration (40\%) and grinding (27\%) are the most common modes of preparation. Skin-related diseases $(78 \%)$ are the most frequent with, followed by those of the Mucosa (12\%). The administration percutaneous (44\%) is the most widely used and then by bath $(21 \%)$.

Conclusion and application: These results show that medicinal plants play a very important role in the treatment of skin diseases and recoverability truly requires the contribution of local knowledge that are owned by the rural populations.

Key words: Ethnobotany, Skin diseases, Medicinal plants, Senegalese, Pharmacopoeia, Bainounk

\section{INTRODUCTION}

Les espèces végétales sont très riches du point de vue de leur nombre et de leur diversité (Spichiger, 2000). Outre leur rôle dans l'équilibre de l'écosystème, les végétaux offrent à l'homme des ressources naturelles indispensables pour sa survie et son développement. Les plantes médicinales constituent un patrimoine précieux pour l'humanité et plus particulièrement pour la majorité des communautés démunies des pays en développement qui en dépendent pour assurer leurs soins de santé primaires et leurs subsistances (Salhi et al., 2010). La médecine traditionnelle soulage plus de $70 \%$ des populations du tiers-monde (Malaisse, 1992 ) et $80 \%$ des populations africaines selon l'Organisation Mondiale de la Santé (OMS) et Jiofack et al., (2010). Les dermatoses sont considérées comme un ensemble de pathologies dont les symptômes les plus visibles se manifestent au niveau des organes cible notamment la peau, les muqueuses et les phanères. Elles sont plus fréquentes là où les conditions d'hygiène sont déficitaires, et leur survenue semble être renforcée ces derniers temps par la recrudescence des infections opportunistes liées au VIH/SIDA (Mozouloua et al., 2011). L'étude réalisée par Lando et al. (2004) à l'hôpital de Yaoundé a montré que les affections dermatologiques sont fréquentes au cours de l'infection par le virus de l'immunodéficience

\section{MATERIEL ET METHODES}

Zone d'étude : Les enquêtes ont été menées dans la Basse Casamance précisément dans le village de Djibonker qui se situe à $12^{\circ} 31^{\prime}$ Latitude Nord et à $16^{\circ} 20^{\prime}$ Longitude Ouest, à $10 \mathrm{~km}$ de Ziguinchor, environ $455 \mathrm{~km}$ humaine $(\mathrm{VIH})$. Les dermatoses constitueraient aussi un problème réel de santé publique dans le monde et dans les pays tropicaux où elles représentent $30 \%$ des consultations en milieu rural (Basset et al., 1999). Au Sénégal, les dermatoses sont très fréquentes. Dans la région de Ziguinchor, elles occupent la $3^{\mathrm{e}}$ place après les principales maladies telles que le paludisme et les affections respiratoires (M. M. Diop, Région Médicale de Ziguinchor, communication personnelle, 03 mai 2013). Djibonker est peuplé essentiellement de Bainounk. Ce groupe ethnique, considéré comme les autochtones de la Casamance, possèderait un savoir traditionnel inestimable véhiculé particulièrement par la langue Bainounk qui est classée aujourd'hui parmi les langues en danger selon School of Oriental and African Studies(SOAS). En plus, la végétation y est fortement menacée ces dernières années du fait de l'intensification des activités anthropiques. Au regard de ces menaces, il est donc impératif de procéder au recueil des savoirs traditionnels détenus par cette population. Les études ethnobotaniques que nous menons depuis 2010 auprès des Bainounk de Djibonker s'inscrivent dans ce cadre et visent une meilleure connaissance de la diversité des plantes médicinales et singulièrement dans cette étude les remèdes utilisés contre les dermatoses.

de Dakar. Ce village fait partie de la communauté rurale d'Enampor qui se trouve dans l'arrondissement de Nyassia dans le département de Ziguinchor (Figure 1). 


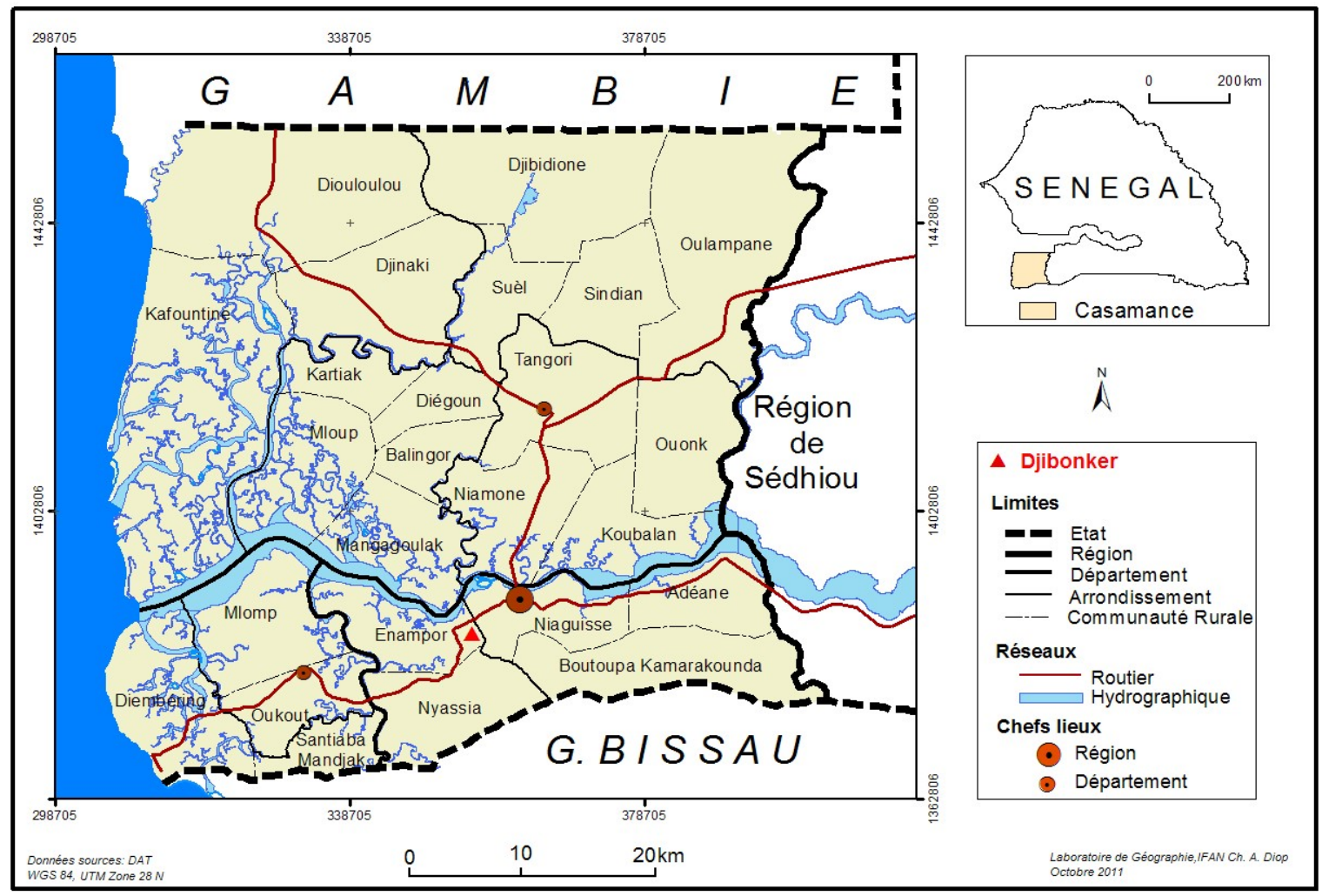

Figure 1 : Carte de localisation de Djibonker, région de Ziguinchor (Sénégal).

La Basse Casamance jouit d'un climat de type tropical qui appartient au domaine guinéo-libérien. Le climat est caractérisé par une longue saison sèche du mois d'octobre à mai, et une saison des pluies qui dure quatre mois et demi avec des quantités de pluies annuelles variant entre 1200 et $1800 \mathrm{~mm}$. La température moyenne annuelle est de $26,7^{\circ} \mathrm{C}$. Les mois les plus chauds sont mai et juin avec des températures moyennes de $28,5^{\circ} \mathrm{C}$ (Berghen et al., 1999). Pendant la saison sèche, la région est parcourue par l'Alizé maritime, vent humide non générateur de pluie. Pendant la saison des pluies, c'est la Mousson qui domine la zone. II s'agit d'un vent chaud et humide générateur des précipitations (Le Pavoux, 2008). La structure géologique et géomorphologique de la Basse Casamance est relativement simple. En effet, on observe des dunes littorales traversées de dépressions humides, de grandes plaines inondables, habituellement aménagées en rizières, des terres sablonneuses soit marécageuses, soit bien drainées, cultivées ou boisées. La végétation est caractérisée par des forêts denses et claires où l'on note la présence d'un grand nombre d'arbres et d'arbustes feuillés durant toute l'année (Berghen et al., 1999).
Collecte des données : La technique des entretiens ouverts semi structurés (Weller, Romney, 1998) a été utilisée pour recueillir les informations sur le terrain. Elle est un outil participatif, permettant de renseigner sur l'utilisation des espèces. Les enquêtes ont été réalisées à l'aide d'un guide d'entretien comportant des questions sur le nom local de l'espèce, les organes utilisés, leurs modes de préparation et d'administration et les parties atteintes par les dermatoses. Les observations directes et conversations occasionnelles sont aussi exploitées à la manière de Martin (2004). Les personnes interrogées sont âgées de 80 et 30 ans dont des tradipraticiens. Nous nous sommes attachés les services d'un guide-interprète comprenant le Gubaher qui est la variante de la langue Bainounk parlée à Djibonker, et qui connaît bien les espèces végétales de la zone d'étude. Le choix du guideinterprète et des personnes interrogées, a été fait avec l'aide de la population locale en tenant compte de leurs connaissances de la flore locale et de leurs usages. Dans de pareils cas, les seuls critères culturellement opérant pour la valeur d'un informateur sont le poids relatif de son savoir face à celui des autres membres de la communauté et/ou la réputation dont il jouit (Grenand et al., 2004). Les entretiens sont réalisés au cours de 
promenades en forêt comme le suggère Cunningham (2002). Ainsi, les espèces sont directement indiquées et récoltées immédiatement. Si l'informateur est trop âgé ou occupé, après l'entretien, nous nous sommes appuyés sur les connaissances du guide-interprète pour récolter des échantillons et, un second passage est effectué chez l'informateur pour une validation des récoltes.

Identification des espèces : Certaines espèces inventoriées sont identifiées sur le terrain. D'autres au laboratoire à l'aide des flores (Lisowski, 2009 a et b ; Berhaut, 1967) et d'autres ouvrages dont Hawthorne et Jongkind(2006) et Arbonnier (2000). Parfois nous avons procédé à une comparaison avec des échantillons

\section{RESULTATS}

Les espèces végétales inventoriées sont réparties en trente-six genres et vingt-cinq familles. Les familles ayant la plus grande diversité spécifique sont les Apocynaceae, les Papilionaceae et les Rubiaceae avec chacune trois espèces, les Caesalpiniaceae, les Euphorbiaceae, les d'herbier de la collection de l'IFAN, l'une des plus importantes d'Afrique de l'Ouest. Après identification les noms valides des taxa sont actualisés à l'aide de la base de données des plantes à fleurs d'Afrique.

L'analyse des données : L'indice de fidélité (Fidelity Level : FL) qui est le pourcentage d'informateurs ayant cité l'usage d'une espèce donnée dans le traitement des dermatoses est calculé selon la technique de Begossi (1996) et Trotter et Logan (1986).

\section{FL $(\%)=(I p / l u) \times 100$}

$>$ Ipest le nombre d'informateurs ayant affirmé l'emploi d'une espèce donnée pour traiter des dermatoses ;

$>$ luest le nombre total d'informateurs interrogés.

Meliaceae, les Moraceae, les Rutaceae et les Sterculiaceae avec chacune deux espèces. Toutes les autres familles sont représentées par une seule espèce (Tableau 1).

Tableau 1 : Liste des plantes utilisées contre les dermatoses chez les Bainounk de Djibonker, région de Ziguinchor (Sénégal).

\begin{tabular}{|l|l|c|}
\hline Taxon & Famille & Indice de fidélité (FL\%) \\
\hline Agave sp & Agavaceae & 55,6 \\
\hline Sorindeia juglandifolia (A.Rich.) Planch. ex Oliv. & Anacardiaceae & 27,8 \\
\hline Annona senegalensisPers. & Annonaceae & 50 \\
\hline Holarrhena floribunda( G.Don) T.Durand\&Schinz & Apocynaceae & 38,9 \\
\hline Saba senegalensis (A.DC.) Pichon & Apocynaceae & 61,1 \\
\hline Voacanga africana Stapf & Apocynaceae & 27,8 \\
\hline Amorphophallus consimilis Blume & Araceae & 33,3 \\
\hline Mikani acordata var. chevalieriC.D.Adams & Asteraceae & 44,4 \\
\hline New bouldia laevis (P.Beauv.) Seem. ex Bureau & Bignoniaceae & 88,9 \\
\hline Adansonia digitata L. & Bombacaceae & 27,8 \\
\hline Mezoneuron benthamianum Baill. & Caesalpiniaceae & 22,2 \\
\hline Detarium senegalensis J.F. Gmel & Caesalpiniaceae & 5,6 \\
\hline Ritchiea capparoides(Andrews) Britten & Capparidaceae & 72,2 \\
\hline Neocarya macrophylla (Sabine) Prance & Chrysobalanaceae & 50 \\
\hline Ipomoea mauritiana Jacq. & Convolvulaceae & 16,7 \\
\hline Alchornea cordifolia(Schumach. \&Thonn.) Müll.Arg. & Euphorbiaceae & 33,3 \\
\hline Jatropha curcas L. & Euphorbiaceae & 66,7 \\
\hline Afzelia Africana Sm. ex Pers. & Fabaceae & 61,1 \\
\hline Crotalaria retusa L. & Fabaceae & 33,3 \\
\hline Indigofera tinctoria L. var. tinctoria & Fabaceae & 11,1 \\
\hline Lawsonia inermis L. & Lithraceae & 22,2 \\
\hline Carapa spp. & Meliaceae & 77,8 \\
\hline Khaya senegalensis(Desr.) A. Juss. & Meliaceae & 94,4 \\
\hline & & \\
\hline
\end{tabular}


Diatta et al J. Appl. Biosci. 2013. Les plantes médicinales utilisées contre les dermatoses dans la pharmacopée Baïnounk de Djibonker, Sénégal

\begin{tabular}{|l|l|c|}
\hline Dichrostachys cinerea(L.) Wight \& Arn. & Mimosaceae & 11,1 \\
\hline Antiaris toxicari avar. africana Scott-Elliot ex A.Chev & Moraceae & 16,7 \\
\hline Ficus thonningii Blume & Moraceae & 44,4 \\
\hline Clematis hirsuta Guill. \& Perr. & Ranunculaceae & 5,6 \\
\hline Garden iatriacantha DC. & Rubiaceae & 27,8 \\
\hline Mitracarpus hirtus(L.) DC. & Rubiaceae & 44,4 \\
\hline Morinda geminata DC. & Rubiaceae & 16,7 \\
\hline Citrus limon (L.) Burm.f. & Rutaceae & 11,1 \\
\hline Zanthoxylum leprieuri Guill. \&Perr. & Rutaceae & 11,1 \\
\hline Allophylus africanus P.Beauv. & Sapindaceae & 11,1 \\
\hline Solanum cerasiferum Dunal & Solanaceae & 77,8 \\
\hline Cola cordifolia (Cav.) R.Br. & Sterculiaceae & 33,3 \\
\hline Waltheria indica L. & Sterculiaceae & 55,6 \\
\hline
\end{tabular}

L'analyse de l'indice de fidélité montre que Khaya senegalensis $(94,4 \%)$, New bouldia laevis $(88,9 \%)$, Carapa spp. $(77,8 \%)$ et Solanum cerasiferum $(77,8 \%)$ sont les espèces ayant les plus grands indices. A l'opposé, Detariumsenegalense et Clematishirsuta ont les plus faibles indices (5,4\% chacune) suivies de Indigofera tinctoria , Dichrostachys cinerea, Citrus limon, Zanthoxylum leprieuri et de Allophylus africanus toutes $11,1 \%$ chacune (Tableau 1).

Les types morphologiques recensés sont dominés par les arbres avec Treize espèces soit $36 \%$ suivis des arbustes avec onze espèces soit $30 \%$ puis les lianes et les herbes avec chacune 6 espèces soit 17\% (Figure2). Les feuilles sont les organes les plus utilisés avec $46 \%$, suivies des racines avec $21 \%$ puis les écorces avec $14 \%$ et le reste avec au plus de 7\% (Figure 3). Différents modes de préparation et d'administration sont utilisées : la macération ( $40 \%$ ) et le broyage ( $27 \%$ ) sont les modes de préparation les plus utilisés viennent ensuite la décoction $(11 \%)$, le chauffage $(9 \%)$ et la poudre, la calcination et le grattage avec chacun 4\% (Figure 4). L'administration percutanée (46\%) est plus fréquente suivie de la boisson $(33 \%)$ et de la prise de bains (21\%) (Figure 5). Les maladies de la peau sont les plus fréquentes avec $(78 \%)$ suivies celles de la muqueuse (12\%) et enfin celles des phanères (10\%) (Figure 6).

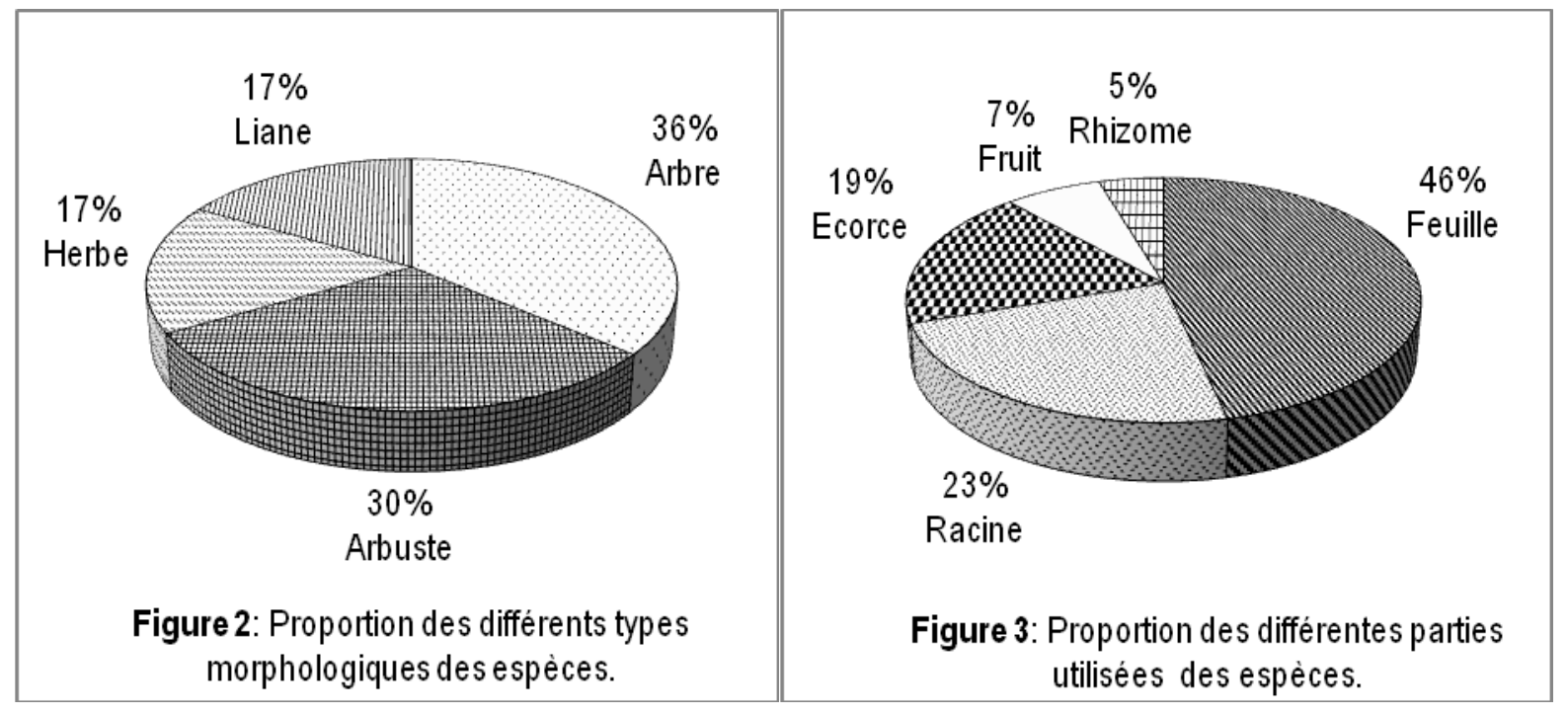




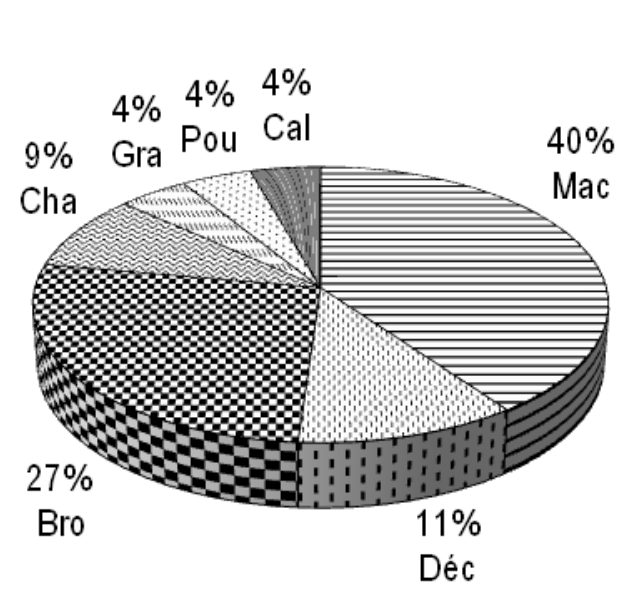

Figure 4: Proportion des différents modes de préparation des espèces.

Figure 5: Proportion des différents modes d'administration des espèces

Bro : Broyage, Cal : Calcination, Cha : Chauffage ; Bai : Bain, Boi : Boisson, Per : Percutanée, Déc : Décoction, Gra : Grattage, Mac : Macération; Pou : Poudre.

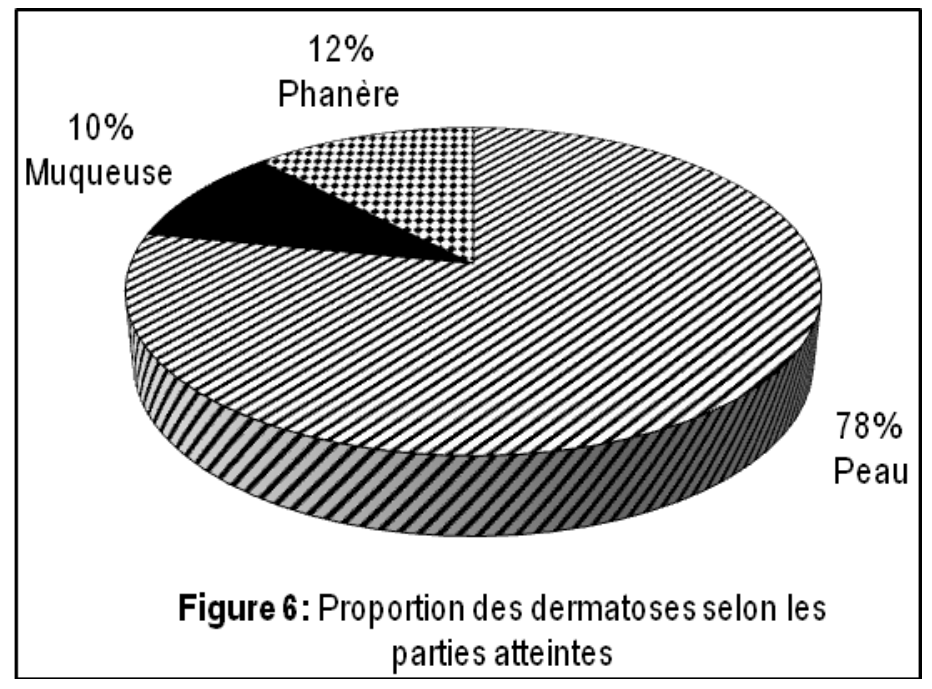

\section{DISCUSSION}

Cette enquête réalisée à Djibonker, nous a permis d'identifier trente-six espèces de plantes médicinales utilisées dans le traitement traditionnel des dermatoses chez les Bainounk Gubaher. Parmi les types morphologiques rencontrés, les arbres sont les espèces les plus utilisées contre les dermatoses suivis des arbustes puis les herbes et les lianes. Ces résultats reflètent la végétation de la zone d'étude dans la basse Casamance où les arbres sont plus représentatifs (Berghen et al., 1999). Les espèces signalées dans la pharmacopée sénégalaise représentent $33,33 \%$ des espèces recensées. Parmi ces espèces certaines sont antidermatosiques et d'autres sont utilisées dans le traitement d'autres pathologies. En effet, Annona senegalensis, Mikania cordata, et New bouldia laevis sont indiquées comme étant des espèces antidermatosiques par Kerharo et al. (1975). Ils montrent aussi qu'Agave sp., Holarhena floribunda, Voacanga africana sont des espèces utilisées dans le traitement des maladies antidiurétiques et Adansonia digitata comme antiasthmatiques. Des études similaires menées à Bangui par Mozoulouaet al. (2011) confirment l'usage d'A. senegalensis, Jatropha curcas et Mitracarpus hirtus contre les dermatoses. En plus, A. senegalensis et $J$. 
curcas sont recommandées contre la constipation (Gueye et al., 2012). Ceci confirme le constat de l'immense variété thérapeutique d'une plante médicinale pour diverses pathologies. L'analyse de l'indice de fidélité montre que Khaya senegalensis, New bouldia laevis, Carapa spp et Solanum cerasiferum sont les mieux connues dans le traitement des dermatoses et seraient sans doute parmi les plus efficaces comme le suggère Gueye et al. (2012). L'efficacité de ces espèces a été démontrée par d'autres d'études. En effet, Lompo et al.(1998) attestent que K. senegalensis est utilisée au Burkina Faso par les tradithérapeutes dans les cas d'inflammations diverses. Les résultats de leur étude ont montré que les extraits organiques de l'écorce de cette espèce ont un effet inhibiteur sur les œdèmes. Au niveau de l'Hôpital Traditionnel de KeurMassar (HTKM) de Dakar ce sont les propriétés antibiotiques de $N$. Laevis qui sont mises en avant Bâ (2012). Selon Da Silva et al.(2009), I'huile de Carapa possède des propriétés thérapeutiques identifiées par la recherche ethno-pharmaceutique : restructuration de l'épiderme, anti-inflammatoire, antiseptique et cicatrisant entre autres. L'huile de Carapa est réputée pour son action réparatrice des lésions cutanées. Ces résultats rejoignent ceux de Gueye et al. (2010) qui indiquent que l'huile de Carapa, est le produit le plus exploité de la plante et son usage médicinal contre les dermatoses est remarquable. Detarium senegalense, Clematis hirsuta, Indigofera tinctoria, Dichrostachys cinerea, Citrus limon, Zanthoxylum leprieuri et Allophylus africanus ont les plus faibles indices de fidélité. Cela prouve que l'usage de ces espèces dans le traitement des dermatoses est méconnu de la majorité des personnes interrogées et, elles ne seraient prescrites que par les tradithérapeutes. Cependant il serait illusoire de penser que ces espèces sont moins efficaces. Bien au contraire, il ne s'agirait plutôt d'un savoir détenu que par certaines personnes compte tenu du mode de dévolution de ce savoir. Les feuilles sont les organes les plus utilisés, suivies des racines, puis les écorces. Ces résultats confirment les études de Mozoulouaet al. (2011) qui ont montré que les feuilles sont les organes végétaux les plus utilisés $(95,23 \%)$ dans le traitement des dermatoses à Bangui confortant ainsi Gueye et al.,(2012) dans le traitement de la constipation. A contrario Teklehaymanot (2009) et Sani (2009) démontrent respectivement que les racines et les écorces sont les

\section{CONCLUSION}

Cette étude a montré que trente-six espèces sont utilisées contre les dermatoses par les Bainounk de organes les plus utilisés en médicine traditionnelle. Pour traiter ces pathologies, différents modes de préparation et d'administration sont utilisées : la macération et le broyage sont les modes de préparation les plus utilisés dans le traitement des dermatoses à Djibonker. II faut signaler que les feuilles bien qu'elles soient les plus utilisées sont préparées de diverses façons. Tous les modes de préparation sont employés pour les feuilles, avec une nette prédominance de la macération. Contrairement à Mozoulouaet al. (2011) qui ont montré que les feuilles sont les organes végétaux les plus utilisés mais sous forme de décocté. Gueye et al., (2012) notent également une forte tendance à l'utilisation de la décoction à chaque fois que les feuilles étaient utilisées contre la constipation. La décoction permettrait de recueillir le plus de principes actifs et atténuerait ou annulerait l'effet toxique de certaines recettes (Salhi et al., 2010). Selon Bitsindou, (1986), la fréquence d'utilisation élevée des feuilles, est due à l'aisance et à la rapidité de la récolte. Mais aussi, au fait qu'elles sont le siège de la photosynthèse et parfois du stockage des métabolites secondaires responsables des propriétés de la plante (Bigendako-Polygenis et Lejoly, 1990). Les autres modes de préparation à savoir le grattage, la poudre, le chauffage, la calcination etc. sont rarement utilisés. Selon les organes atteints (la peau, la muqueuse et les phanères), les dermatoses sont subdivisées en trois types de pathologies : Les maladies de la peau sont les plus fréquentes à Djibonker suivies celles de la muqueuse et enfin celles des phanères. Ces résultats peuvent être expliqués par le fait que la peau est un organe fin et fragile couvrant la totalité du corps. Ainsi, elle subit de multiples agressions par son contact permanent avec le milieu extérieur (Sorg, 2010). L'administration percutanée est plus fréquente suivie de la prise de bains. Ceci montre un lien direct entre les types de dermatoses rencontrées et les modes d'administration. Elle indiquerait aussi que les dermatoses sont bien connues de la médecine traditionnelle Bainounkau regard des modes d'administration qu'elle recommande. Mais cela indique une complexité des propriétés médicinales que contiennent ces espèces qui mériteraient beaucoup plus d'attention dans la recherche des alternatives thérapeutiques dans le monde.

Djibonker. Les espèces les plus utilisées sont Khaya senegalensis, New bouldia laevis, Carapa spp et 
Solanum cerasiferum surtout dans le cas des dermatoses liées à la peau qui sont prédominantes. Parmi les organes utilisés on note une nette prédominance des feuilles. Les modes de préparation sont multiples mais la macération est plus courante. L'administration des médicaments traditionnels proposés se fait surtout par voie percutanée. Les remèdes traditionnels indiqués dans cette étude présentent un intérêt réel dans la prise en charge thérapeutique en milieu rural. En effet, il est apparu que les guérisseurs traditionnels participent ici à

\section{REMERCIEMENTS}

Nous remercions l'ensemble de nos informateurs et toute la population Gubaher (Bainounk de Djibonker). Merci à VolkswagenStiftung (Fondation Volkswagen) qui a bien

\section{REFERENCES}

Arbonnier M, 2000. Arbres, Arbustes et Lianes des Zones Sèches d'Afrique de l'Ouest. Ed. CIRADMNHN-UICN, Paris, 542p.

Bâ D, 2012. La chronique du mardi au jardin botanique: le Newbouldia laevis. Disponible à l'adresse http://www.seyilaabehtkm.com/2012 12 01 archive.html. (Consulté le 30 juin 2013)

Basset D, Pratolon F, Ravel C, Pyechberty JJ, Dereure J et Dedet J, 2001. Les leishmantases déclarées en France en 1999. Bul. Épidémiol. Hebdo.,5: 1-7.

Begossi A, 1996. Use of ecological methods in ethnobotany : Diversity indices. Ecological Methods in Ethnobotany, 50: 280-289.

Berghen CV et Manga A, 1999. Introduction à un voyage en Casamance. Enampor, un village de riziculteurs en Casamance, au Sénégal. Paris : L'Harmattan, 14,15 et 17p.

Berhaut J, 1967. Flore du Sénégal plus Complète avec les Forêts Humides de la Casamance. Edn. Clair Afrique ; 485p.

Bigendako-Polygenis MJ \&Lejoly J, 1990. La pharmacopée traditionnelle au Burundi. Pesticides et médicaments en santé animale. Pres. Univ. Namur. Pp. 425-442.

Bitsindou M, 1986. Enquête sur la phytothérapie traditionnelle à Kindamba et Odzala (Congo) et analyse de convergence d'usage des plantes médicinale en Afrique centrale.Mem. Doc (inéd.). Univ. Libre de Bruxelles. 482 pp.

CJB, 2012. Base de données des plantes d'Afrique. Site: http://www.ville- la résolution d'un problème majeur de santé que sont les dermatoses. Au cours de nos entretiens, nous avons noté que les Gubaher (Bainounk), ont une grande connaissance des plantes médicinales surtout celles prescrites contre les dermatoses, si bien qu'aujourd'hui, elles devraient jouer un grand rôle dans la résolution des problèmes de santé si la médecine traditionnelle était d'une part reconnue au Sénégal et prise en compte dans les politiques de santé des pays en développement.

voulu financer le projet «Pots, Plantes et peuple : une documentation interdisciplinaire des savoirs Bainounk » (DoBeS 3P).

ge.ch/musinfo/bd/cjb/africa/recherche.php?langu $\mathrm{e}=\mathrm{fr}$ (Consulté le 02 Février 2013).

Cunningham AB, 2002. Applied ethnobotany: People, wild plant use and conservation. People and Conservation Manuel, Earth scan publications Ltd, 300p.

Da Silva VP, Oliveira RR et Figueiredo MR, 2009. Isolation of limonoids from seeds of Carapaguianensis Aublet (Meliaceae) by highspeed countercurrent chromatography. Phytochem Anal., 20(1):77-81.

Grenand $P$, Moretti C, Jacquemin H et Prévost MF, 2004. Pharmacopées traditionnelles en Guyane. Ed. IRD Paris, France, 816p.

Gueye M, Cisse A, Diatta CD, Diop S et Koma S, 2012. Étude ethnobotanique des plantes utilisées contre la constipation chez les Malinké de la communauté rurale de Tomboronkoto, Kédougou (Sénégal). Int. J. Biol. Chem. Sci. 6(2): 778-779.

Gueye M, Kenfack D \& Forget PM, 2010. Importance socio-culturelle, potentialités économiques et thérapeutiques du Carapa(Meliaceae) au Sénégal. In : X. van der Burgt, J. van der Maesen\& J.-M. Onana (eds), Systématique et Conservation des Plantes Africaines, pp. 359367.

Hawthorne W et Jongkind C, 2006. Woody plants of western African forests:a guide to the forest trees, shrubs and lianes from Senegal to Ghana. Ed. Royal Botanic Gardens, Kew, 1023p.

Jiofack T, Fokunang C, Guedje NM, Kemeuze V, Fongnzossie $\mathrm{E}$, Nkongmeneck BA, 
Mapongmetsem PM, Tsabang N, 2010. Ethnobotanical uses of medicinal plants of two ethnoecological regions of Cameroon. International Journal of Medicine and Medical Sciences 2 (3): 60-79.

Kerharo J, Bouquet A et Debray M, 1975.Médecines et pharmacopées traditionnelles du Sénégal, du Congo et de Madagascar, Études Médicales, (1) $88 \mathrm{p}$.

Lando MJ, Mboua JN, Tardy M, Noumsi N, NzeuseuVetKouanfack C, 2004. Affections cutanéomuqueuses au cours de l'infection au VIH /SIDA. Mother and Child HealthClinics Vol. 1(3) 2004: 158-165.

Le Pavoux S, 2008. La forêt de Kanoufa : une forêt à protéger. Rapport de stage du BTSA gestion et protection de la nature, Spécialité gestion des espaces naturels. $61 \mathrm{p}$.

Lisowski S, 2009a. Flore (Angiospermes) de la République de Guinée, permière partie (texte). ScriptaBotanicaBelgica, vol 41, Ed. National Botanic Garden of Belgium, Meise, 517P.

Lisowski S, 2009b. Flore (Angiospermes) de la République de Guinée, deuxième partie (illustrations). ScriptaBotanicaBelgica, vol 42, Ed. National Botanic Garden of Belgium, Meise, $566 \mathrm{p}$.

Lompo M, Ouedraogo S, Sourabie S et Guissou IP, 1998. KASE: Valorisation d'une plante médicinale anti-inflammatoire. Pharm. MM. Trad. Afr., $10:$ :68-79.

Malaisse $F, 1992$. La gestion des produits sauvages comestibles, Défis- Sud, $7:$ 18-19.

Martin GJ, 2004. Ethnobotany: A methods manual. Earthscan Publications, London.

Mozouloua D, Apema AKR et Nguengue JP, 2011. Étude préliminaire des plantes médicinales à effets antidermatosiques utilisées en pharmacopée à Bangui. URSAD. 3,5 et $6 p$.
Teklehaymanot T, 2009. Ethnobotanical study of knowledge and medicinal plants use by the people in Dek Island in Ethiopia.Journal of Ethnopharmacology, 124: 69-78.

Trotter RT, Logan MH, 1986.Informant Consensus : a New Approach for Identifying Potentially Effective Médicinal Plants. Edn. BedforeHills : New York ; 91-112.

Sani RA, 2009. Caractérisation biophysique des ressources ligneuses dans un site reverdi et un site dégrade dans le département de Mirriah. Mémoire de fin de cycle en vue de l'obtention du diplôme d'Ingénieur, option : eaux et forêts. Université Abdou Moumouni, Faculté d'Agronomie, p42.

Salhi S, Fadli M, Zidane L \& Douira A, 2010. Études floristique et ethnobotanique des plantes médicinales de la ville de Kénitra (Maroc). Lazaroa 31: 133-146.

Sorg 0, 2010. La dermatotoxicologie : une discipline à l'avenir prometteur pour l'étude des effets sur la peau des nouveaux xénobiotiques. Rev Med Suisse, 6: 879-881.

Spichiger RE, Savolanen VV, Figeat M. 2000.Botanique Systématique des Plantes à Fleurs. Une Approche Phylogénétique Nouvelle des Angiospermes des RégionsTempérées et Tropicales. Presses Polytechniques et Universitaires Romandes ; Lausanne (Suisse) xii $+372 p$.

Srajbf, 2012. La peau. Site http://www.srajbf.org/pdf/la peau.pdf (Consulté le 30 juin 2013)

Weller S C, Romney A K, 1998. Systematic Data Collection. Qualitative Research Methods Series, 10.SAGE. Publications, Newbury Park, USA. 